\title{
The effects of the physiognomic stimuli taketa and maluma on the meanings of neutral stimuli
}

\author{
MARTIN S. LINDAUER \\ State University of New York, College at Brockport, Brockport, New York
}

\begin{abstract}
In physiognomy, sensory, perceptual, and affective connotations are suggested by an object. For example, a mountain, in addition to being literally big, may also seem "quiet, looming, and threatening." The capacity of physiognomically endowed but meaningless stimuli (like taketa and maluma) to transfer these meanings to similarly unfamiliar but neutral stimuli was examined on 15 perceptual, affective, and sensory rating scales $(N=118)$. The meanings of the two neutral stimuli were influenced in 26 instances (vs. 8 cases in which the neutral stimuli followed one other); most changes were affective and sensory rather than perceptual; and the shifts in meanings were noncongruent-taketa's "aggressiveness," for example, led to a "peaceful" rating of the two neutral shapes. The implications of these findings are discussed.
\end{abstract}

The term physiognomy is usually applied to persons, referring mainly to the expressiveness of their faces, but it also includes the evocative qualities of all kinds of objects (Arnheim, 1972; Gombrich, 1972; Kohler, 1938/1947; Lindauer, 1984a; Pratt, 1969; Schlesinger, 1980; Werner, 1956; Werner \& Wapner, 1952; Winner, 1982). For example, not only a face but also a mountain can seem “quiet, dark, and peaceful."' Sensory, perceptual, and affective physiognomic connotations are also evoked by nonsense stimuli. For example, an unknown round shape may be seen and felt to be "peaceful" (Figure 1-1), whereas an unfamiliar angular form may appear "aggressive" (Figure 1-2). Furthermore, the sight of these shapes can have sensory or synesthetic properties: The shapes called maluma and taketa "sound" as if they should be given these nonsense names, respectively (Fox, 1935; Marks, 1978).

The advantage of studying physiognomy in unfamiliar nonsense shapes is that past experience, learning, and memory have little if any effect on the response. Consequently, structural features inherent in the stimulus (Gibson, 1979) can be demonstrated without their being complicated by obvious associations; hence the impressiveness of changes in color, size, and distance in taketa and maluma, which have been found to differ in these respects from one another and from other nonsense but physiognomically neutral stimuli (Lindauer, 1986, 1988). For example, taketa was judged to be bigger and farther away, as well as more saturated and brighter in color than it actually was, in relation to maluma, a random-dot pattern, and an amoeba-like form (Figures 1-3 and 1-4, respectively). Subsequent studies, which have included ratings, have shown that heightened sensory, perceptual, and affective meanings characterize taketa and maluma and

Correspondence may be addressed to Martin S. Lindauer, now at the Institute of Gerontology, The University of Michigan, 300 North Ingalls, Ann Arbor, MI 48109-2007. not the neutral stimuli, and that the specific meanings of the two physiognomic stimuli on these dimensions differ from each other and from those of the other stimuli (Lindauer, 1990).

To ascertain whether the sensory, perceptual, and affective meanings of the physiognomic stimuli would transfer to neutral stimuli was the main purpose of the present study. It was predicted that the physiognomic stimuli would change the meanings of the neutral stimuli that followed them, that there would be as many sensory and perceptual changes as affective ones, and that these changes would be congruent with the meanings of the physiognomic stimuli. For example, the aggressive taketa should increase the aggressive meaning of the neutral dot pattern and the amorphous shape. It was also expected that taketa, as a more distinctive stimulus than maluma (Lindauer, 1986, 1988,1990 ), would have a stronger influence. Finally, the neutral stimuli would also have some transfer effect on each other (but less than the physiognomic stimuli would), since all stimuli carry some physiognomic meanings (Lindauer, 1990). The effects of the two neutral stimuli would be observed when each was preceded by each of the physiognomic stimuli, the other neutral stimulus, or no stimulus. The meanings of all stimuli were known from previous research (Lindauer, 1990).

\section{METHOD}

Two neutral and two physiognomic stimuli (Figure 1) were rated on 15 affective, perceptual, and sensory scales. The same materials had been used earlier to determine the meanings of the stimuli (Lindauer, 1990). (They had been presented randomly, rather than systematically, as in this study.) In four experimental conditions, each neutral stimulus was preceded by one of the two physiognomic stimuli, either taketa or maluma. In four control conditions, each neutral stimulus was rated either by itself or when it was preceded by the other neutral stimulus. (The latter condition determined the effect of one neutral stimulus on the other.)

The meanings of the physiognomic and neutral stimuli were known and distinguished from one another (Lindauer, 1990). For example, taketa was perceptually bright, and maluma was nearer than the others; taketa was also affectively aggressive, while maluma was peaceful; and in sen- 


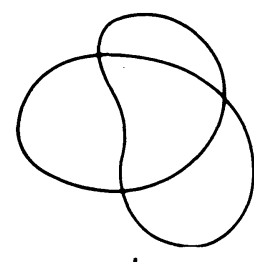

I
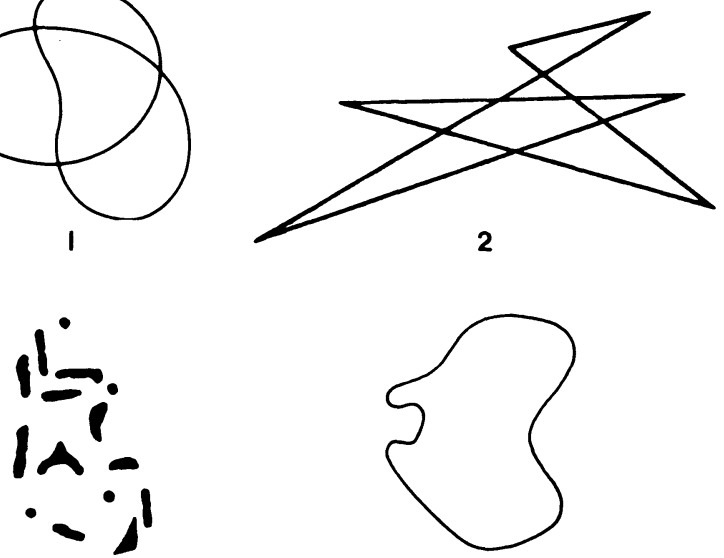

3

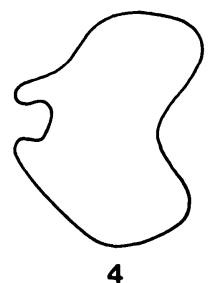

Figure 1. The four stimuli used: physiognomic (maluma and taketa, Nos. 1 and 2, respectively) and neutral (dot pattern and amoebalike shape, Nos. 3 and 4 , respectively).

sory terms, taketa was noisy, while maluma was quiet. As for the neutral stimuli, although they had fewer distinctive meanings than did the physiognomic stimuli, there were still some: The shape was perceptually pale, whereas the dots were dark; and the shape was affectively weak and sensorily slow.

Each of the eight conditions was represented by a booklet. Its cover, the same in all conditions, stated the purpose of the study (i.e., "this is a way of measuring the meanings of unfamiliar stimuli''), and included instructions on how to use the scales, along with three scales to be used for rating a sample stimulus (not used in the actual study). The subjects were told that they could not return to a previous page once a page had been turned, that they should not skip any of the scales, and that they should work at their own pace. The purpose of the study was explained during debriefing at the end, as an examination of the expressiveness of physiognomic and neutral stimuli. The subjects' responses were anonymous.

A single stimulus shape, one of four, was located at the top of the page; each was the same size $(6.80 \mathrm{~cm}$ in height). Below the stimulus were 15 bipolar adjective pairs arranged in one of four random orders; the poles were reversed in two of the orders. The same scales were used for all stimuli. A blank sheet was inserted between the pages of the booklets to mask the second stimulus (if present).

The booklets were distributed to small groups of subjects (3-5 at a time), at either the beginning or the end of the class, and monitored. The subjects were volunteers (for credit) from several undergraduate psychology classes (49\% were from the introductory courses); most were women (64\%), and nearly all were $17-22$ years old. There were 13-16 subjects in each condition (total $N=118$ ). Those who had previously taken a perception course, or who had participated in earlier studies with these stimuli, were excused. The subjects were told that some of the booklets differed, and that some people would finish at different times. The control conditions, which contained only one stimulus, took about $5 \mathrm{~min}$ to complete, whereas the others required $10-15 \mathrm{~min}$.

\section{RESULTS}

\section{Physiognomic Effects on the Neutral Dot Pattern}

The affective and sensory meanings of the dot pattern differed across all the conditions $[F(3,105-106)=8.30$ and 8.23 , respectively, $p<.01$ ]; the perceptual meanings did not differ $(F<1)$. Consider first the differences between the stimuli on the affective scales $[F(12,424)=$ $10.89, p<.01]$. (Here and elsewhere, unless otherwise noted, Newman-Keuls analyses were used, $p<.05$ ). When taketa was shown first, the dot pattern was rated as more peaceful, relaxed, and tender, as well as weaker and friendlier, than it was when the two other neutral stimuli or no stimuli were initially presented. And when maluma was shown first, the dots were rated as stronger and unfriendlier than when they were followed by the other neutral stimuli or none $[F(3,461) \geq 5.51, p<.01$, for both these comparisons].

The changed meanings of the neutral stimuli were opposite to the meanings of the physiognomic stimuli (Lindauer, 1990). For example, the earlier presence of the aggressive taketa and the weak maluma resulted in the dot pattern's being rated more peaceful and stronger, respectively, than would otherwise have been the case.

Prior exposure to the physiognomic stimuli also influenced the sensory meanings of the dot pattern $[F(12,420)=$ $4.69, p<.01]$. When preceded by taketa, compared to the other (or no) stimuli, the dots were rated as slower, hotter, quieter, and blunter; and when preceded by maluma, the dots were rated sharper.

Paralleling the affective ratings, exposure to the physiognomic stimuli had an opposite influence on the meanings of the dots. (There was one exception: Taketa had not been previously distinguished as "hot.") The neutral shape, however, also had an effect on the dots, although less than did the physiognomic stimuli: The ratings of the dots became noisier and sharper, compared to their meanings when the dots followed the physiognomic stimuli $[F(3,481) \geq 2.71, p<.04$, for these comparisons]. However, the shape had not been previously shown to be distinguished by any of these meanings.

On the perceptual scales, there was a significant interaction $[F(12,428)=2.25, p<.01]$. The dot stimulus was rated as paler when it was preceded by taketa (previously characterized as a bright stimulus) than when it was preceded by the other stimuli $[F(3,535)=2.18$, $p=.09]$. The dots were also rated as nearer when they were shown alone than when they were preceded by maluma and the other stimuli $[F(3,535)=2.46$, $p=.06]$. Maluma had earlier been distinguished by its near meanings, the neutral shape by its distance.

Similar differences between the stimuli, especially for taketa, were also found on the overall affective and sensory categories, in which the set of five scales was combined. Across the affective scales, the dots preceded by taketa received significantly lower scores $(M=3.23)$, than they did when they were preceded by maluma $(M=4.79)$, the neutral shape $(M=4.74)$, or no stimulus $(M=4.37)$. And on the sensory category, when the dots were preceded by taketa, they received significantly lower ratings $(M=$ $3.31)$ than they did when they were preceded by maluma $(M=4.31)$, the neutral shape $(M=4.37)$, or no stimulus $(M=3.78)$.

In summary, when taketa was shown first, the meanings of the dot stimulus were influenced in 11 instances, compared to four changes when maluma preceded the dots, and three after exposure to the neutral shape. More 
changes were found on the affective and sensory scales (7 each) than on the perceptual scales (2). Furthermore, 12 of the 15 shifts in the meaning of the neutral stimulus were the opposite of those which described the physiognomic stimuli in an earlier study (Lindauer, 1990). And of the three shifts in meaning that were due to the presence of another neutral stimulus (the ambiguous shape), only one was in the opposite direction to its meaning, while the other two had not been distinguishable attributes in the earlier study.

\section{Physiognomic Effects on the Neutral Shape}

The affective meanings of the neutral shape were influenced by the preceding stimulus conditions $[F(3,97)=$ $6.95, p<.01]$, and these depended on the stimuli and scales involved $[F(12,388)=3.63, p<.01]$. The shape preceded by taketa was more peaceful, relaxed, friendly, and tender-meanings that were the opposite of taketa's. Although the same shifts were also found when the shape was preceded by the neutral dots, none of these had distinguished the dots' meaning earlier (Lindauer, 1990). Maluma also had an effect on the shape, making it more unfriendly in meaning (in contrast with maluma's "friendliness"). [The specific differences noted above were based on $F(3,402) \geq 3.43, p<.05$.]

With respect to the sensory scales $[F(3,96)=6.07$, $p<.01$ ], differences were found on three: fast-slow $[F(3,474)=2.94, p<.05]$, quiet-noisy $[F(3,474)=$ $6.06, p<.01]$, and light-heavy $[F(3,474)=5.34$, $p<.01]$. A significant interaction $[F(12,384)=2.69$, $p<.01]$ indicated the following: The shape preceded by maluma was noisier, and when preceded by taketa, it was quieter; and the shape preceded by maluma was faster than the shape rated alone, and slower when it was preceded by taketa. These effects on the shape, like most of those already noted, were the opposite of the physiognomic stimuli's known meanings. A quiet and light (in weight) shift in the meanings of the shape also occurred when it was preceded by the neutral dots, a change unrelated to the dots' own meanings (determined in Lindauer, 1990).

There was less of an effect on the perceptual scales $[F(3,97)=2.39, p>.05]$, which was also true for the neutral dot pattern above: The shape was rated as smaller when it was alone, compared to when it was preceded by all three stimuli $[F(3,482)=2.74, p<.04]$. This effect is the opposite of taketa's and the dots' meanings.

The stimuli also differed on the combined affective and sensory categories of meaning but not, as above, on the perceptual category. In each case, maluma led to higher affective and sensory scores for the shape, compared to the presence of the other (or no) stimuli ( $M=3.45 \mathrm{vs.}$ 2.66-3.06, $p<.05$ ).

In summary, the neutral shape's meaning was influenced in 18 instances (the same number was also found for the neutral dots). Prior exposure to taketa and to the dots influenced the shape's meanings in seven cases each, while maluma had an effect in four cases. The physiognomic stimuli therefore had less of an effect on the shape than they had on the dots; and the neutral dot stimulus had more of an effect on the neutral shape than the reverse. Nine of the 11 effects due to the physiognomic stimuli were the opposite of their original meanings. (The two exceptions, both involving maluma, were on scales on which it had not been previously distinguished.) But only two of the five changes brought about by the dots' presence were the opposite of their meaning. (These changes were not associated with the dots' distinguishable meanings.) The affective meanings were influenced the most (in nine cases), followed by the sensory (in six cases) and the perceptual (in three cases). The relative ranking of these changes, in which affective and sensory effects were greater than the perceptual ones, parallels those found for the neutral dots.

\section{The Effects of the Two Physiognomic Stimuli on Both Neutral Stimuli}

Combining the results for each of the neutral stimuli treated separately above, the following patterns emerge: Previous exposure to the two physiognomic stimuli shifted the meanings of the two neutral stimuli in 26 instances. Taketa led to more changes than did maluma, 18 versus 8 ; and of these 18 shifts, 11 were influences on the dot stimulus, most of them (9) on affective meanings. The two neutral stimuli also affected each other's meanings in 10 instances; the dots accounted for 7 of these. The physiognomic stimuli led to 21 opposite changes in the meanings of the neutral stimuli (of which 16 were due to taketa). In contrast, the neutral stimuli led to an opposite change in meanings in only 3 cases; the only instance in which a change was congruent occurred when maluma shifted the "near" meaning of the dots. Both the dot and the shape stimuli were influenced the same number of times (18). The affective meanings were changed more than any other category (18), followed by the sensory (13) and the perceptual (7).

\section{DISCUSSION}

The physiognomic stimuli maluma and taketa did change the affective, sensory, and perceptual meanings of the neutral stimuli that followed them. But not all three types of meanings were equally susceptible to change. Affective ratings were influenced somewhat more than sensory ones, and both were changed considerably more than the perceptual values. Although the three kinds of physiognomic meanings are equally potent, with respect to the physiognomic stimuli themselves (Lindauer, 1990), their influence on other stimuli is not the same.

Affect may therefore be physiognomy's most potent characteristic, in terms of its generalizability to other stimuli. Affect has been given a preeminent position by many writers (Schlesinger, 1980; Stein, 1975; Werner, 1956; Werner \& Wapner, 1952), although others in different contexts have emphasized more of its perceptual (Arnheim, 1972; Ryan, 1938) and perhaps synesthetic properties (Fox, 1935; Marks, 1978).

Physiognomy may transfer its affective context to subsequent stimuli in accordance with a "top-down"' process (Lazarus, 1982; Zajonc, 1980), in which sensory and perceptual processing sufficient for registration occurs, but not enough for conscious identification (Rock, 1984). For example, the "unfeeling" quality of a person's face influences the meaning of what that person says or does. These events, in sensory terms, are interpreted as fitting someone who is "as hard as a rock"; and in perceptual terms, unyielding and resistant qualities are demonstrated. 
These attributions, however, did not appear to be self-consciously or deliberately made (i.e., cognitively constructed), at least on the basis of spontaneous self-reports and the postexperimental debriefing. However, it is difficult to distinguish between different processes on a phenomenological basis. It would be equally difficult to maintain that the meanings of the nonsense stimuli changed because the physiognomic stimuli reminded subjects of what they had in common. The stimuli were equally unfamiliar (Lindauer, 1986). And if the subjects were guessing, their responses should have been random.

Another possibility is that the expressive properties that define physiognomy are inherent in the stimulus (Gibson, 1979). A structural explanation, with its focus on the nature of the stimulus, fits the phenomenological reports (Lindauer, 1984a): The qualities of the stimuli were assigned immediately and spontaneously. Physiognomy as an intrinsic quality of the stimulus helps account for other findings, such as how similar meanings can be assigned to unknown or unfamiliar objects across diverse cultures (Asch, 1955; Osgood, May, \& Miron, 1975) and among very young children (Greenauer \& Lindauer, 1981; Wallach \& Kogan, 1965). Physiognomic connotations that go beyond the given also help account for how apparently unrelated but familiar stimuli (like colors, stick figures, days of the week, and smells) can be matched with one another (Leijonhielm, 1967; Rosenberg \& Langer, 1969).

Puzzling, however, was the "contrary effect," in which the meanings of the neutral stimuli were changed so as to be the opposite of those of the preceding physiognomic stimuli. This may have been the result of an adaptation-level response (Helson, 1964) to the surprise felt when an ostensibly meaningless figure provoked an unaccountable meaning. To illustrate, consider the impact of taketa: Here is an unknown shape that nevertheless evoked an unexpected meaning of "aggressiveness,", an experience without any readily apparent basis. As a consequence, an opposite (nonconscious) reaction could have occurred, one of "friendliness" for the following neutral stimulus. The initially strange and puzzling effect was compensated for, "neutralized," or balanced by an opposite response.

A contextual effect also occurs with meaningful stimuli. Consider a face that appears to be aggressive (Ekman, 1973); this same meaning may, but need not, transfer to the hand gestures that accompany it. In one context, as when one is in love, the hands can be judged as friendly (and the face only playfully aggressive). Another example of an "opposite effect" occurs when a smiling stranger reaches out toward a child, and the apparently friendly face is interpreted as hostile in intent.

Thus, in both person and object perception, expressive physiognomic qualities can have both a congruent and a noncongruent effect, depending on the context. Physiognomic juxtapositions of stimuli that either do or do not "fit"' have similarly dramatic effects in literature (Tytler, 1982; Winner, 1982) and art (Amheim, 1972; Gombrich, 1972; Lindauer, 1969, 1970a, 1970b, 1984b).

\section{REFERENCES}

ARnheIm, R. (1972). The Gestalt theory of expression. In R. Arnheim (Ed.), Toward a psychology of art: Collected essays (pp. 51-73). Berkeley, CA: University of California Press.

Asch, S. E. (1955). On the use of the metaphor in the description of persons. In H. Werner (Ed.), On expressive language (pp. 29-38). Worcester, MA: Clark University.

Ekman, P. (1973). Darwin and facial expression: A century of research in review. New York: Academic Press.

Fox, C. W. (1935). An experimental study of meaning. American Journal of Psychology, 43, 545-579.

GiBSON, J. J. (1979). The ecological approach to perception. Boston: Houghton Mifflin.

Gombrich, E. H. (1972). The mask and the face: The perception of physiognomic likeness in life and art. In E. H. Gombrich, J. Hochberg,
\& M. Black (Eds.), Art, perception and reality (pp. 1-46). Baltimore: Johns Hopkins University Press.

Greenauer, M., \& Lindauer, M. S. (1981). Physiognomic perception of positive and negative stimuli among five-year old children. ERIC Resources in Education, PS 021 417/ED 206416.

Helson, H. (1964). Adaptation-level theory. New York: Harper \& Row. KOHLER, W. (1947). Gestalt psychology. New York: Liveright. (Original work published 1938)

LAZARUS, R. S. (1982). Thoughts on the relations between emotion and cognition. American Psychologist, 37, 1019-1024.

Leuonhielm, C. (1967). Colours, forms and art. Stockholm: Almquist \& Wiksell.

Lindauer, M. S. (1969). The orientation of form in abstract art. Proceedings of the 7th Annual Convention-APA, 4, 475-476.

Lindauer, M. S. (1970a). Physiognomic properties of form perception in abstract art and titles. Proceedings of the 78th Annual Convention-APA, 5, 493-494.

Lindauer, M. S. (1970b). Psychological aspects of form perception in abstract art. Science de l'Art, 7, 19-24.

Lindauer, M. S. (1984a). Physiognomic perception. In R. J. Corsini (Ed.), Wiley encyclopedia of psychology (Vol. 3, pp. 34-35). New York: Wiley.

Lindauer, M. S. (1984b). Physiognomy and art: Approaches from above, below, and sideways. Visual Arts Research, 10, 52-65.

LINDAUER, M. S. (1986). Perceiving, imaging, and preferring the colors of physiognomic stimuli. American Journal of Psychology, 99, 233-255.

LindAuer, M. S. (1988). Size and distance perception of the physiognomic stimulus "taketa." Bulletin of the Psychonomic Society, 26, 217-220.

LINDAUER, M. S. (1990). The meanings of the physiognomic stimuli taketa and maluma. Bulletin of the Psychonomic Society, 28, 47-50.

MARKs, L. E. (1978). The unity of the senses. New York: Academic Press.

Osgood, C. E., May, W. H., \& Miron, M. S. (1975). Cross-cultural universals of affective meaning. Urbana: University of Illinois Press.

Pratt, C. C. (1969). Introduction to The task of Gestalt psychology (pp. 3-29), by W. Kohler. Princeton, NJ: Princeton University Press.

Rock, I. (1984). Perception. New York: Scientific American.

Rosenberg, B. C., \& LANGer, J. (1969). Nonverbal learning. American Journal of Psychology, 82, 481-490.

RYAN, T. A. (1938). Dynamic, physiognomic, and other neglected properties of perceived objects: A new approach to comprehending. American Journal of Psychology, 51, 629-649.

SChlesinger, L. B. (1980). Physiognomic perception: Empirical and theoretical perspectives. Genetic Psychology Monographs, 101, 71-97.

Stein, M. L. (1975). Physiognomic cue test. New York: Behavioral Publications.

Tytler, G. (1982). Physiognomy in the European novel. New York: Scribner.

Wallach, M., \& KogaN, N. (1965). Modes of thinking in young children. New York: Holt, Rinehart \& Winston.

WerNer, H. (1956). On physiognomic perception. In G. Kepes (Ed.), The new landscape in art and science (pp. 280-282). Chicago: Paul Theobald.

WERNER, H., \& WAPNER, S. (1952). Toward a general theory of perception. Psychological Review, 59, 324-338.

WINNER, E. (1982). Invented worlds: The psychology of the arts. Cambridge, MA: Harvard University Press.

ZaJONC, R. B. (1980). Feeling and thinking: Preferences need no references. American Psychologist, 35, 151-175.

(Manuscript received July 10, 1989.) 